\title{
Bounds for stochastic processes on product index spaces
}

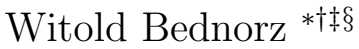 \\ September 6, 2018
}

\begin{abstract}
In this paper we discuss the question how to bound supremum of a stochastic process with the index set of a product type. There is a tempting idea to approach the question by the analysis of the process on each of the marginal index spaces separately. However it turns out that we also need to study suitable partitions of the whole index space. We show what can be done in this direction and how to use the method to reprove some known results. In particular we observe that all known applications of the Bernoulli Theorem can be obtained in this way, moreover we use the shattering dimension to slightly extend the application to VC classes. We also show some application to the regularity of paths for processes which take values in vector spaces. Finally we give a short proof of the Mendelson-Paouris result on sums of squares for empirical processes.
\end{abstract}

\section{Introduction}

In this paper $I$ denotes a countable set and $(F,\|\cdot\|)$ a separable Banach space. Consider the class $\mathcal{A}$ of subsets of $I$. We say that the class $\mathcal{A}$ satisfies the maximal inequality if for any symmetric independent random variables $X_{i}, i \in I$ taking values in $F$ the following inequality holds

$$
\mathbf{E} \sup _{A \in \mathcal{A}}\left\|\sum_{i \in A} X_{i}\right\| \leqslant K \mathbf{E}\left\|\sum_{i \in I} X_{i}\right\|,
$$

where $K$ depends on $\mathcal{A}$ only. We stress that in this paper $K$ will be used to denote constants that appear in the formulation of our results and may depend on their structure. We use $c, C, L, M$ to denote absolute constants which may change their values from line to line by numerical factors. Also we write $\sim$ to express that two quantities are comparable up to a universal constant. This will help us to reduce the notation applied in this paper. It is an easy observation to see that (1.1) is equivalent to

$$
\mathbf{E} \sup _{A \in \mathcal{A}}\left\|\sum_{i \in A} v_{i} \varepsilon_{i}\right\| \leqslant K \mathbf{E}\left\|\sum_{i \in I} v_{i} \varepsilon_{i}\right\|,
$$

\footnotetext{
*Subject classification:Primary 60G15; Secondary 60G17

${ }^{\dagger}$ Keywords and phrases: $\mathrm{VC}$ classes, shattering dimension, stochastic inequalities

¥Institute of Mathematics, University of Warsaw, Banacha 2, 02-097 Warsaw, Poland

$\S$ Research partially supported by MNiSW Grant N N201 608740 and Mobility Plus
} 
where $\left(v_{i}\right)_{i \in I}$, consists of vectors in $F$ and $\left(\varepsilon_{i}\right)_{i \in I}$ is a Bernoulli sequence, i.e. a sequence of independent r.v.'s such that $\mathbf{P}\left(\varepsilon_{i}= \pm 1\right)=\frac{1}{2}$.

To understand what is the right characterization of such classes $\mathcal{A}$ we have to recall the notion of $\mathrm{VC}$ dimension. We say that $\mathcal{A}$ has $\mathrm{VC}$ dimension $d$ if there exists a set $B \subset I,|B|=d$ such that $|\{B \cap A: \quad A \in \mathcal{A}\}|=2^{d}$ but for all $B \subset I,|B|>d,|\{B \cap A: A \in \mathcal{A}\}|<2^{d+1}$. It means that $\mathcal{A}$ shatters some set $B$ of cardinality $d$ but do not shatter any set of cardinality $d+1$. The result which has been proved in [1] as a corollary of the Bernoulli Theorem states that finite $\mathrm{VC}$ dimension is the necessary and sufficient condition for the class $\mathcal{A}$ to have the property (1.1). Since our paper strongly refers to the Bernoulli Theorem we recall its formulation. We start from mentioning Talagrand's result for Gaussian's processes. In order to find two-sided bounds for supremum of the process $G(t)=\sum_{i \in I} t_{i} g_{i}$, where $t \in T \subset \ell^{2}(I)$ and $\left(g_{i}\right)_{i \in I}$ is a Gaussian sequence, i.e. a sequence of independent standard Gaussian r.v.'s we need Talagrand's $\gamma_{2}(T)$ numbers, cf. Definition 2.2.19 in [15] or (3.1) below. By the well known Theorem 2.4.1 in [15] we have

$$
\mathbf{E} \sup _{t \in T} G(t) \sim \gamma_{2}(T) .
$$

The Bernoulli Theorem, i.e. Theorem 1.1 in [1, concerns a similar question for processes of random signs.

Theorem 1.1 Suppose that $T \subset \ell^{2}(I)$. Then

$$
\mathbf{E} \sup _{t \in T} \sum_{i \in I} t_{i} \varepsilon_{i} \sim \inf _{T \subset T_{1}+T_{2}}\left(\sup _{t \in T_{1}}\|t\|_{1}+\gamma_{2}\left(T_{2}\right)\right) .
$$

where the infimum is taken over all decompositions $T_{1}+T_{2}=\left\{t_{1}+t_{2}: t_{1} \in\right.$ $\left.T_{1}, t_{2} \in T_{2}\right\}$ that contain the set $T$ and $\|t\|_{1}=\sum_{i \in I}\left|t_{i}\right|$.

Note that if $0 \in T$ then we can also require that $0 \in T_{2}$ in the above result. The consequence of Theorem 1.1 to our problem with maximal inequalities is as follows.

Theorem 1.2 The class $\mathcal{A}$ satisfies (1.1) with a finite constant $K$ if and only if $\mathcal{A}$ is a $V C$ class of a finite dimension. Moreover the square root of the dimension is up to a universal constant comparable with the optimal value of $K$.

Observe that part of the result is obvious. Namely one can easily show that if $\mathcal{A}$ satisfies the maximal inequality then it is necessarily a $\mathrm{VC}$ class of a finite dimension. Indeed let $\left(\varepsilon_{i}\right)_{i \in I}$ be a Bernoulli sequence. Suppose that set $B \subset I$ is shattered. Let $x_{i}=1$ for $i \in B$ and $x_{i}=0, i \notin B$. Obviously

$$
\mathbf{E}\left|\sum_{i \in I} x_{i} \varepsilon_{i}\right|=\mathbf{E}\left|\sum_{i \in B} \varepsilon_{i}\right| \leqslant \sqrt{|B|}
$$

and on the other hand

$$
\mathbf{E} \sup _{A \in \mathcal{A}}\left|\sum_{i \in A} x_{i} \varepsilon_{i}\right|=\mathbf{E} \sup _{A \in \mathcal{A}}\left|\sum_{i \in A \cap B} x_{i} \varepsilon_{i}\right| \geqslant \mathbf{E} \sum_{i \in B} \varepsilon_{i} 1_{\varepsilon_{i}=1}=|B| / 2 .
$$


Consequently if (1.1) holds then $K \geqslant \sqrt{|B|} / 2$. Therefore (1.1) implies that cardinality of $B$ must be smaller or equal $4 K^{2}$.

Much more difficult is to prove the converse statement, i.e that for each $\mathrm{VC}$ class $\mathcal{A}$ of dimension $d$ the inequality (1.2) holds with $K$ comparable with $\sqrt{d}$. In order to prove the result one has to first replace the basic formulation of the maximal inequality - (1.2) by its equivalent version

$$
\mathbf{E} \sup _{A \in \mathcal{A}} \sup _{t \in T}\left|\sum_{i \in A} t_{i} \varepsilon_{i}\right| \leqslant K \mathbf{E} \sup _{t \in T}\left|\sum_{i \in I} t_{i} \varepsilon_{i}\right|,
$$

where $\left(\varepsilon_{i}\right)_{i \in I}$ is a Bernoulli sequence and $0 \in T \subset \ell^{2}(I)$. Note that we use absolute values since part of our work concerns complex spaces. However it is important to mention that in the real case $\mathbf{E} \sup _{t \in T} \sum_{i \in I} t_{i} \varepsilon_{i}$ is comparable with $\mathbf{E}_{\sup _{t \in T}}\left|\sum_{i \in I} t_{i} \varepsilon_{i}\right|$ if $0 \in T$ and therefore we often require in this paper that $0 \in T \subset \ell^{2}(I)$. Let us denote

$$
b(T)=\mathbf{E} \sup _{t \in T}\left|\sum_{i \in I} t_{i} \varepsilon_{i}\right|, g(T)=\mathbf{E} \sup _{t \in T}\left|\sum_{i \in I} t_{i} g_{i}\right|,
$$

where $\left(\varepsilon_{i}\right)_{i \in I},\left(g_{i}\right)_{i \in I}$ are respectively Bernoulli and Gaussian sequence. We recall that, what was known for a long time [5, 7, (1.4) holds when Bernoulli random variables are replaced by Gaussians, i.e.

$$
\mathbf{E} \sup _{A \in \mathcal{A}} \sup _{t \in T}\left|\sum_{i \in A} g_{i} t_{i}\right| \leqslant C \sqrt{d} \mathbf{E} \sup _{t \in T}\left|\sum_{i \in I} t_{i} g_{i}\right|=C \sqrt{d} g(T),
$$

for any $0 \in T \subset \ell^{2}(I)$. Due to Theorem 1.1 one can cover the set $T$ by $T_{1}+T_{2}$, where $0 \in T_{2}$ and

$$
\max \left\{\sup _{t \in T_{1}}\|t\|_{1}, g\left(T_{2}\right)\right\} \leqslant L b(T) .
$$

Therefore using (1.5) and (1.6)

$$
\begin{aligned}
& \mathbf{E} \sup _{A \in \mathcal{A}} \sup _{t \in T}\left|\sum_{i \in A} \varepsilon_{i} t_{i}\right| \\
& \leqslant \sup _{t \in T_{1}}\|t\|_{1}+\mathbf{E} \sup _{A \in \mathcal{A}} \sup _{t \in T_{2}}\left|\sum_{i \in A} \varepsilon_{i} t_{i}\right|
\end{aligned}
$$

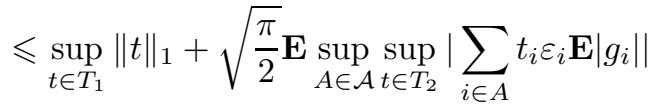

$$
\begin{aligned}
& \leqslant \sup _{t \in T_{1}}\|t\|_{1}+\sqrt{\frac{\pi}{2}} \mathbf{E} \sup _{A \in \mathcal{A}} \sup _{t \in T_{2}}\left|\sum_{i \in A} t_{i} g_{i}\right| \\
& \leqslant \sup _{t \in T_{1}}\|t\|_{1}+\sqrt{\frac{\pi}{2}} g\left(T_{2}\right) \leqslant C L \sqrt{d} b(T) .
\end{aligned}
$$

This proves Theorem 1.2

We show another example in which a similar approach works. Let $G$ be a compact Abelian group and $\left(v_{i}\right)_{i \in I}$ a sequence of vectors taking values in $F$. 
Let $\chi_{i}, i \in I$ be characters on $G$. The deep result of Fernique 4 is

$$
\mathbf{E} \sup _{h \in G}\left\|\sum_{i \in I} v_{i} \chi_{i}(h) g_{i}\right\| \leqslant C\left(\mathbf{E}\left\|\sum_{i \in I} v_{i} g_{i}\right\|+\sup _{\left\|x^{*}\right\| \leqslant 1} \mathbf{E} \sup _{h \in G}\left|\sum_{i \in I} x^{*}\left(v_{i}\right) \chi_{i}(h) g_{i}\right|\right) .
$$

This can be rewritten similarly as (1.5), i.e. for any $0 \in T \subset \ell^{2}(I)$ (which is a complex space in this case)

$$
\mathbf{E} \sup _{h \in G} \sup _{t \in T}\left|\sum_{i \in I} t_{i} \chi_{i}(h) g_{i}\right| \leqslant C\left(g(T)+\sup _{t \in T} \mathbf{E} \sup _{h \in G}\left|\sum_{i \in I} t_{i} \chi_{i}(h) g_{i}\right|\right) .
$$

Once again the Bernoulli Theorem allows to prove a similar result for Bernoulli sequences. Namely by Theorem 1.1 we get the decomposition $T \subset T_{1}+T_{2}$, $0 \in T_{2}$ such that

$$
\max \left\{\sup _{t \in T_{1}}\|t\|_{1}, g\left(T_{2}\right)\right\} \leqslant L b(T) .
$$

Consequently using (1.7), (1.8) and $\left|\chi_{i}(h)\right| \leqslant 1$ we get

$$
\begin{aligned}
& \operatorname{Esup}_{h \in G} \sup _{t \in T}\left|\sum_{i \in I} t_{i} \chi_{i}(h) \varepsilon_{i}\right| \\
& \leqslant \sup _{t \in T_{1}}\|t\|_{1}+\mathbf{E} \sup _{h \in G} \sup _{t \in T_{2}}\left|\sum_{i \in A} \varepsilon_{i} t_{i} \chi_{i}(h)\right| \\
& \leqslant \sup _{t \in T_{1}}\|t\|_{1}+\sqrt{\frac{\pi}{2}} \mathbf{E} \sup _{h \in G} \sup _{t \in T_{2}}\left|\sum_{i \in I} t_{i} \chi_{i}(h) g_{i}\right| \\
& \leqslant \sup _{t \in T_{1}}\|t\|_{1}+C\left(g\left(T_{2}\right)+\sup _{t \in T_{2}} \mathbf{E} \sup _{h \in G}\left|\sum_{i \in I} t_{i} \chi_{i}(h) g_{i}\right|\right) \\
& \leqslant C L\left(b(T)+\sup _{t \in T_{2}} \mathbf{E} \sup _{h \in G}\left|\sum_{i \in I} t_{i} \chi_{i}(h) g_{i}\right|\right) .
\end{aligned}
$$

The final step is the Marcus-Pisier estimate [12] (see Theorem 3.2.12 in [15])

$$
\sup _{t \in T_{2}} \mathbf{E} \sup _{h \in G}\left|\sum_{i \in I} t_{i} \chi_{i}(h) g_{i}\right| \leqslant M \sup _{t \in T_{2}} \mathbf{E} \sup _{h \in G}\left|\sum_{i \in I} t_{i} \chi_{i}(h) \varepsilon_{i}\right| .
$$

Note that (1.11) is deeply based on the translational invariance of the distance

$$
d_{t}(g, h)=\left(\sum_{i \in I}\left|t_{i}\right|^{2}\left|\chi_{i}(g)-\chi_{i}(h)\right|^{2}\right)^{\frac{1}{2}} g, h \in G .
$$


Since we may assume that $T_{2} \subset T-T_{1}$ we get

$$
\begin{aligned}
& \sup _{t \in T_{2}} \mathbf{E} \sup _{h \in G}\left|\sum_{i \in I} t_{i} \chi_{i}(h) \varepsilon_{i}\right| \\
& \leqslant \sup _{t \in T} \mathbf{E} \sup _{h \in G}\left|\sum_{i \in I} t_{i} \chi_{i}(h) \varepsilon_{i}\right|+\sup _{t \in T_{1}} \mathbf{E} \sup _{h \in G}\left|\sum_{i \in I} t_{i} \chi_{i}(h) \varepsilon_{i}\right| \\
& \leqslant \sup _{t \in T} \mathbf{E} \sup _{h \in G}\left|\sum_{i \in I} t_{i} \chi_{i}(h) \varepsilon_{i}\right|+L b(T) .
\end{aligned}
$$

Combining (1.10) with (1.11) and (1.13) we get the following result.

Theorem 1.3 Suppose that $0 \in T \subset \ell^{2}(I)$. For any compact group $G$ and $a$ collection of vectors $v_{i} \in F$ in a complex Banach space $(F,\|\cdot\|)$ and characters $\chi_{i}$ on $G$ the following holds

$$
\mathbf{E} \sup _{h \in G} \sup _{t \in T}\left|\sum_{i \in I} t_{i} \chi_{i}(h) \varepsilon_{i}\right| \leqslant K\left(b(T)+\sup _{t \in T} \mathbf{E} \sup _{h \in G}\left|\sum_{i \in I} t_{i} \chi_{i}(h) \varepsilon_{i}\right|\right) .
$$

The aim of this note is to explore questions described above in a unified language. Simply we consider random processes $X(u, t),(u, t) \in U \times T$ with values in $\mathbb{R}$ or $\mathbb{C}$, which means we study stochastic processes defined on product index sets. In particular we cover all canonical processes in this way. Indeed, suppose that $U \subset \mathbb{R}^{I}$ or $\mathbb{C}^{I}$ and $T \subset \mathbb{R}^{I}$ or $\mathbb{C}^{I}$ are such that for any $a \in U$ and $t \in T$ we have that $\sum_{i \in I}\left|u_{i} t_{i}\right|^{2}<\infty$. Then for any family of independent random variables $X_{i}$ such that $\mathbf{E} X_{i}=0, \mathbf{E}\left|X_{i}\right|^{2}=1$,

$$
X(u, t)=\sum_{i \in I} u_{i} t_{i} X_{i}, \quad u \in U, t \in T
$$

is a well defined process. As we have mentioned our main classes of examples concern Gaussian canonical processes where $X_{i}=g_{i}, i \in I$ are standard normal variables or Bernoulli canonical processes where $X_{i}=\varepsilon_{i}, i \in I$ are random signs. In particular we aim to find bounds for $\mathbf{E} \sup _{u \in U}\left\|\sum_{i \in I} u_{i} v_{i} \varepsilon_{i}\right\|$, where $v_{i} \in F, i \in I$, formulated in terms of $\mathbf{E}\left\|\sum_{i \in I} v_{i} \varepsilon_{i}\right\|$. One of the results we state is the application of the shattering dimension introduced by Mendelson and Vershynin 14, which enable us to generalize Theorem 1.2. In this way we deduce that under mild conditions imposed on $\mathcal{A} \subset \mathbb{R}^{I}$ we have

$$
\mathbf{E} \sup _{u \in U}\left\|\sum_{i \in I} u_{i} v_{i} X_{i}\right\| \leqslant K \mathbf{E}\left\|\sum_{i \in I} v_{i} X_{i}\right\|
$$

for any independent symmetric r.v.'s $X_{i}, i \in I$. We show how to apply the result in the analysis of convex bodies and their volume in high dimensional spaces. On the other hand we can use the approach to study processes $X(t)=\left(X_{i}(t)\right)_{i \in I}$, $t \in[0,1]$ which take values in $\mathbb{R}^{I}$ or $\left.\mathbb{C}^{I}\right)$. For example to check whether paths $t \rightarrow X(t)$ belong to $\ell^{2}$ we should consider

$$
X(u, t)=\sum_{i \in I} u_{i} X_{i}(t), u=\left(u_{i}\right)_{i \in I} \in U, t \in[0,1],
$$


where $U$ is the unit ball in $\ell^{2}(I)$, i.e. $U=\left\{u \in \mathbb{R}^{I}: \sum_{i \in I}\left|u_{i}\right|^{2} \leqslant 1\right\}$. The finiteness of $\|X(t)\|_{2}<\infty$ is equivalent to the finiteness of $\sup _{u \in u}|X(u, t)|$. Similarly we can treat a well known question in the theory of empirical processes. Suppose that $(\mathcal{E}, \mathcal{B})$ is a measurable space and $\mathcal{F}$ a countable family of measurable real functions on $\mathcal{E}$. Let $X_{1}, X_{2}, \ldots, X_{N}$ be independent random variables which take values in $(\mathcal{E}, \mathcal{B})$, we may define

$$
X(u, f)=\sum_{i=1}^{N} u_{i} f\left(X_{i}\right), \quad u=\left(u_{i}\right)_{i=1}^{N} \in U, f \in \mathcal{F},
$$

where $U=B_{N}(0,1)=\left\{u \in \mathbb{R}^{N}: \sum_{i=1}^{N}\left|u_{i}\right|^{2} \leqslant 1\right\}$. Then it is clear that

$$
\sup _{u \in U}|X(u, f)|^{2}=\sum_{i=1}^{N}\left|f\left(X_{i}\right)\right|^{2}, \quad \text { for all } f \in \mathcal{F} .
$$

In the last section we give a short proof of Mendelson-Paouris result [13 that gives an upper bound for $\mathbf{E} \sup _{u \in U} \sup _{f \in \mathcal{F}}|X(u, t)|$.

\section{Upper bounds}

For the sake of completeness we give an idea how to bound stochastic processes. The approach we present slightly extends former results of Latala 9, 10 and Mendelson-Paouris [13. Suppose that $\mathbf{E}|X(t)-X(s)|<\infty$ for all $s, t \in T$. For each $s, t \in T$ and $n \geqslant 0$ we define $\bar{q}_{n}(s, t)$ as the smallest $q>0$ such that

$$
\begin{aligned}
& F_{q, n}(s, t)=\mathbf{E} q^{-1}(|X(t)-X(s)|-q)_{+} \\
& =\int_{1}^{\infty} \mathbf{P}(|X(t)-X(s)|>q t) d t \leqslant N_{n}^{-1} .
\end{aligned}
$$

We prove the following observation.

Lemma 2.1 Function $\bar{q}_{n}(s, t), s, t \in T$ is a distance on $T$, namely is symmetric, satisfies the triangle inequality and $\bar{q}_{n}(s, t)=0$ if and only if $X(s)=X(t)$ a.s.

Proof. Obviously $\bar{q}_{n}(s, t)$ is finite and symmetric $\bar{q}_{n}(s, t)=\bar{q}_{n}(t, s)$. To see that it equals 0 if and only if $\mathbf{P}(|X(t)-X(s)|>0)>0$ a.s. note that if $X(s) \neq X(t)$ then $\mathbf{E}|X(t)-X(s)|>0$. The function $q \rightarrow F_{q, n}(s, t)$ is decreasing continuous and $F_{q, n}(s, t) \rightarrow \infty$ if $q \rightarrow 0$ and $\mathbf{E}|X(t)-X(s)|>0$. Moreover $F_{q, n}(s, t)$ is strictly decreasing on the interval $\left\{q>0: F_{q, n}(s, t)>0\right\}$ and consequently $\bar{q}(s, t)$ is the unique solution of $F_{q, n}(s, t)=N_{n}^{-1}$, namely

$$
\mathbf{E}\left(\bar{q}_{n}(s, t)\right)^{-1}\left(|X(t)-X(s)|-\bar{q}_{n}(s, t)\right)_{+}=N_{n}^{-1} .
$$

Finally we show that $\bar{q}$ satisfies the triangle inequality. Indeed for any $u, v, w \in T$ either $\bar{q}(u, v)=0$ or $\bar{q}(v, w)=0$ or $\bar{q}(u, w)=0$ and the inequality is trivial or all the quantities are positive and then

$$
F_{\bar{q}_{n}(u, v), n}(u, v)=F_{\bar{q}_{n}(v, w), n}(v, w)=F_{\bar{q}_{n}(u, w), n}(u, w)=N_{n}^{-1} .
$$


It suffices to observe

$$
\begin{aligned}
& \frac{1}{\bar{q}_{n}(u, v)+\bar{q}_{n}(v, w)} \mathbf{E}\left(|X(u)-X(w)|-\bar{q}_{n}(u, v)-\bar{q}_{n}(w, v)\right)_{+} \\
& \leqslant \mathbf{E}\left(\frac{|X(u)-X(w)|+|X(w)-X(v)|}{\bar{q}_{n}(u, v)+\bar{q}_{n}(w, v)}-1\right)_{+} .
\end{aligned}
$$

The function $x \rightarrow(x-1)_{+}$is convex which implies that

$$
(p x+q y-1)_{+} \leqslant p(x-1)_{+}+q(y-1)_{+}
$$

for $p, q \geqslant 0, p+q=1$ and $x, y>0$. We use (2.2) for

$$
x=\frac{|X(u)-X(v)|}{\bar{q}_{n}(u, v)}, y=\frac{|X(v)-X(w)|}{\bar{q}_{n}(v, w)}
$$

and

$$
p=\frac{\bar{q}_{n}(u, v)}{\bar{q}_{n}(u, v)+\bar{q}_{n}(w, v)}, \quad q=\frac{\bar{q}_{n}(v, w)}{\bar{q}_{n}(u, v)+\bar{q}_{n}(w, v)}
$$

Therefore

$$
\begin{aligned}
& \frac{1}{\bar{q}_{n}(u, v)+\bar{q}_{n}(v, w)} \mathbf{E}\left(|X(u)-X(w)|-\bar{q}_{n}(u, v)-\bar{q}_{n}(w, v)\right)_{+} \\
& \leqslant p \mathbf{E}\left(\frac{|X(u)-X(v)|}{\bar{q}_{n}(u, v)}-1\right)_{+}+q \mathbf{E}\left(\frac{|X(v)-X(w)|}{\bar{q}_{n}(v, w)}-1\right)_{+} \\
& \leqslant p N_{n}^{-1}+q N_{n}^{-1}=N_{n}^{-1}
\end{aligned}
$$

which by definition gives that

$$
\bar{q}_{n}(u, v)+\bar{q}_{n}(v, w) \geqslant \bar{q}_{n}(v, w) .
$$

Obviously usually we do not need to use the optimal distances $\bar{q}_{n}$ and replace the construction which is sufficient for our purposes. We say that a family of distances $q_{n}, n \geqslant 0$ on $T$ is admissible if $q_{n}(s, t) \geqslant \bar{q}_{n}(s, t)$ and $q_{n+1}(s, t) \geqslant$ $q_{n}(s, t)$. For example Latala 9, 10 and Mendelson-Paouris 13, used moments, namely if $\|X(t)-X(s)\|_{p}=\left(\mathbf{E}|X(t)-X(s)|^{p}\right)^{\frac{1}{p}}<\infty, p \geqslant 1$ then we may take $q_{n}(s, t)=2\|X(t)-X(s)\|_{2^{n}}$. Indeed observe that

$$
\mathbf{E}\left(\frac{|X(t)-X(s)|}{2\|X(t)-X(s)\|_{2^{n}}}-1\right)_{+} \leqslant \frac{\mathbf{E}|X(t)-X(s)|^{2^{n}}}{\left(2\|X(t)-X(s)\|_{2^{n}}\right)^{2^{n}}} \leqslant \frac{1}{N_{n}} .
$$

Following Talagrand we say that a sequence of partitions $\mathcal{A}_{n}, n \geqslant 0$ of a set $T$ is admissible if it is increasing, $\mathcal{A}_{0}=\{T\}$ and $\left|\mathcal{A}_{n}\right| \leqslant N_{n}$. Let us also define admissible sequences of partitions $\mathcal{A}=\left(\mathcal{A}_{n}\right)_{n \geqslant 0}$ of the set $T$, which means nested sequences of partitions such that $\mathcal{A}_{0}=\{T\}$ and $\left|\mathcal{A}_{n}\right| \leqslant N_{n}$. For each $A \in \mathcal{A}_{n}$ we define

$$
\Delta_{n}(A)=\sup _{s, t \in A} q_{n}(s, t) .
$$

By $A_{n}(t)$ we call devote the element of $\mathcal{A}_{n}$ that contains point $t$. For each $A \in \mathcal{A}_{n}, n \geqslant 0$ we define $t_{A}$ as an arbitrary point in $A$. We may and will 
assume that if $t_{A} \in B \in \mathcal{A}_{n+1}, B \subset A \in \mathcal{A}_{n}$ then $t_{B}=t_{A}$. Let $\pi_{n}(t)=t_{A_{n}(t)}$, then $\pi_{0}(t)=t_{T}$ is a fixed point in $T$. Let $T_{n}=\left\{\pi_{n}(t): t \in T\right\}$ for $n \geqslant 0$. Clearly $T_{n}, n \geqslant 0$ are nested, namely $T_{n} \subset T_{n+1}$ for $n \geqslant 0$. For each stochastic process $X(t), t \in T$ and $\tau \geqslant 0$ we may define

$$
\gamma_{X}^{\tau}(T)=\inf _{\mathcal{A}} \sup _{t \in T} \sum_{n=0}^{\infty} \Delta_{n+\tau}\left(A_{n}(t)\right) .
$$

We prove that for $\tau \geqslant 2, \gamma_{X}^{\tau}(T)$ is a good upper bound for $\mathbf{E}_{\sup _{s, t \in T}} \mid X(t)-$ $X(s) \mid$.

Theorem 2.2 For $\tau \geqslant 2$ the following inequality holds

$$
\mathbf{E} \sup _{s, t \in T}|X(t)-X(s)| \leqslant 4 \gamma_{X}^{\tau}(T) .
$$

Proof. Note that

$$
\begin{aligned}
\left|X(t)-X\left(\pi_{0}(t)\right)\right| \\
\leqslant \sum_{n=0}^{\infty} q_{n+\tau}\left(\pi_{n+1}(t), \pi_{n}(t)\right) \\
+\sum_{n=0}^{\infty}\left(\left|X\left(\pi_{n+1}(t)\right)-X\left(\pi_{n}(t)\right)\right|-q_{n+\tau}\left(\pi_{n+1}(t), \pi_{n}(t)\right)\right)_{+} \\
\leqslant \sum_{n=0}^{\infty} \Delta_{n+\tau}\left(A_{n}(t)\right)+\sum_{n=0}^{\infty} \sum_{u \in T_{n}} \sum_{v \in A_{n}(u) \cap T_{n+1}}\left(|X(u)-X(v)|-q_{n+\tau}(u, v)\right)_{+} .
\end{aligned}
$$

For any $\varepsilon>0$ one can find nearly optimal admissible partition $\left(\mathcal{A}_{n}\right)_{n \geqslant 0}$ such that

$$
\sup _{t \in T} \sum_{n=0}^{\infty} \Delta_{n+\tau}\left(A_{n}(t)\right) \leqslant(1+\varepsilon) \gamma_{X}^{\tau}(T)
$$

and therefore

$$
\begin{aligned}
& \mathbf{E} \sup _{t \in T}\left|X(t)-X\left(\pi_{0}(t)\right)\right| \\
& \leqslant \gamma_{X}^{\tau}(T)+\varepsilon+\sum_{n=0}^{\infty} \sum_{u \in T_{n}} \sum_{v \in A_{n}(u) \cap T_{n+1}} \frac{q_{n+\tau}(u, v)}{N_{n+\tau}} \\
& \leqslant(1+\varepsilon) \gamma_{X}^{\tau}(T)+\sum_{n=0}^{\infty} \sum_{u \in T_{n}} \Delta_{n+\tau}\left(A_{n}(u)\right) \frac{N_{n+1}}{N_{n+\tau}} \\
& \leqslant \gamma_{X}^{\tau}(T)\left(1+\varepsilon+\sum_{n=0}^{\infty} \frac{N_{n} N_{n+1}}{N_{n+\tau}}\right) \\
& \leqslant(1+\varepsilon) \gamma_{X}^{\tau}(T)+\gamma_{X}^{\tau}(T)\left(\frac{N_{0} N_{1}}{N_{2}}+\sum_{n=1}^{\infty} \frac{1}{N_{n}}\right) \\
& \leqslant \gamma_{X}^{\tau}(T)\left(1+\varepsilon+\sum_{n=0}^{\infty} 2^{-n-1}\right)
\end{aligned}
$$


where in the last line we used $N_{n}^{2}=N_{n+1}, N_{n} \geqslant 2^{n+1}$ for $n \geqslant 1$ and $N_{0} N_{1} / N_{2}=$ $1 / 4<1 / 2$. Since $\varepsilon$ is arbitrary small we infer that $\mathbf{E} \sup _{t \in T}\left|X(t)-X\left(\pi_{0}(t)\right)\right| \leqslant$ $2 \gamma_{X}^{\tau}(T)$ and hence $\mathbf{E} \sup _{s, t}|X(t)-X(s)| \leqslant 4 \gamma_{X}^{\tau}(T)$.

The basic question is how to construct admissible sequences of partitions. The simplest way to do this goes through entropy numbers. Recall that for a given distance $\rho$ on $T$ the quantity $N(T, \rho, \varepsilon)$ denotes the smallest number of balls of radius $\varepsilon$ with respect to $\rho$ necessary to cover the set $T$. Consequently for a given $\tau \geqslant 0$ we define entropy numbers as

$$
e_{n}^{\tau}=\inf \left\{\varepsilon>0: N\left(T, q_{n+\tau}, \varepsilon\right) \leqslant N_{n}\right\}, n \geqslant 0 .
$$

Having the construction ready we may easily produce an admissible sequence of partitions. On each level $n$ there exists at most $N_{n}$ sets of $q_{n+\tau}$ diameter $2 \varepsilon$ that covers $T$. To obtain nested sequence of partitions we have to intersect all the sets constructed at levels $0,1, \ldots, n-1$. The partition $\mathcal{A}_{n}$ has no more than $N_{0} N_{1} \ldots N_{n-1} \leqslant N_{n}$ elements. Moreover for each set $A \in \mathcal{A}_{n}$ we have

$$
\Delta_{n+\tau-1}(A) \leqslant 2 e_{n-1}^{\tau} \text { for } n \geqslant 1 .
$$

Obviously $\Delta_{\tau-1}(T) \leqslant \Delta_{\tau}(T) \leqslant 2 e_{0}^{\tau}$. Let $\mathcal{E}_{X}^{\tau}(T)=\sum_{n=0}^{\infty} e_{n}^{\tau}$, then for any $\tau \geqslant 1$

$$
\gamma_{X}^{\tau-1}(T) \leqslant 2 e_{0}^{\tau}+2 \sum_{n=1}^{\infty} e_{n-1}^{\tau} \leqslant 4 \mathcal{E}_{X}^{\tau}(T) .
$$

and hence by Theorem 2.2 with $\tau \geqslant 3$

$$
\mathbf{E} \sup _{s, t \in T}|X(t)-X(s)| \leqslant 16 \mathcal{E}_{X}^{\tau}(T) .
$$

We turn to our main question of processes on product spaces. Consider $X(u, t)$, $u \in U, t \in T$ we define two different families of distances $q_{n, t}$ and $q_{n, u}$ admissible to control marginal processes respectively $u \rightarrow X(u, t)$ on $U$ and $t \rightarrow X(u, t)$ on $T$.

First for a given $t \in T$, let $q_{n, t}, n \geqslant 0$ be a family of distances on $U$ admissible for the process $u \rightarrow X(u, t)$ and let $e_{n, t}^{\tau}, n \geqslant 0$ be entropy numbers on $U$ constructed for $q_{n, t}, n \geqslant 0$. By (2.3) we infer that for $\tau \geqslant 3$

$$
\mathbf{E} \sup _{u, v \in U}|X(u, t)-X(v, t)| \leqslant 16 \sum_{n=0}^{\infty} e_{n, t}^{\tau} .
$$

If we define $\mathcal{E}_{X, t}^{\tau}(U)=\sum_{n=0}^{\infty} e_{n, t}^{\tau}$ and $\mathcal{E}_{X, T}^{\tau}(U)=\sup _{t \in T} \mathcal{E}_{X, t}^{\tau}(U)$ then we may rewrite (2.4) as

$$
\sup _{t \in T} \mathbf{E} \sup _{u, v \in U}|X(u, t)-X(v, t)| \leqslant 16 \mathcal{E}_{X, T}^{\tau}(U),
$$

and in this way the entropy numbers may be used to bound the family of processes $u \rightarrow X(u, t)$ where $t \in T$.

On the other hand for a given $u \in U$ let $q_{n, u}, n \geqslant 0$ be a family of distances on $T$ admissible for $t \rightarrow X(u, t)$. Obviously $q_{n, U}=\sup _{u \in U} q_{n, u}$ is a good upper bound for all distances $q_{n, u}$. Let

$$
\gamma_{X, U}^{\tau}(T)=\inf _{\mathcal{A}} \sup _{t \in T} \sum_{n=0}^{\infty} \Delta_{n+\tau, U}\left(A_{n}(t)\right)
$$


where the infimum is taken over all admissible partitions $\mathcal{A}=\left(\mathcal{A}_{n}\right)_{n \geqslant 0}$ of $T$ and

$$
\Delta_{n, U}(A)=\sup _{s, t \in A} q_{n, U}(s, t)=\sup _{s, t \in A} \sup _{u \in U} q_{n, u}(s, t) .
$$

Theorem 2.2 applied to distances $q_{n, U}, n \geqslant 0$ implies that

$$
\sup _{u \in U} \mathbf{E} \sup _{s, t \in T}|X(u, t)-X(u, s)| \leqslant 4 \gamma_{X, U}^{\tau}(T) .
$$

We prove that the described two quantities i.e. $\mathcal{E}_{X, T}^{\tau}(U)$ and $\gamma_{X, U}^{\tau}(T)$ suffice to control the process $X(u, t), u \in U, t \in T$.

We state our main result which extends the idea described in Theorem 3.3.1 in [15.

Theorem 2.3 For any $\tau \geqslant 4$ the following inequality holds

$$
\mathbf{E} \sup _{u, v \in U} \sup _{s, t \in T}|X(u, t)-X(v, s)| \leqslant 24\left(\gamma_{X, U}^{\tau}(T)+\mathcal{E}_{X, T}^{\tau}(U)\right) .
$$

Proof. We first observe that if $\gamma_{X, U}^{\tau}(T)<\infty$ then, by the definition, for any $\varepsilon>0$ there exists an admissible partition sequence $\mathcal{C}=\left(\mathcal{C}_{n}\right)_{n \geqslant 0}$ of $T$, which satisfies

$$
(1+\varepsilon) \gamma_{X, U}^{\tau}(T) \geqslant \sup _{t \in T} \sum_{n=0}^{\infty} \Delta_{n+\tau, U}\left(B_{n}(t)\right) .
$$

Let us fix $C \in \mathcal{C}_{n}$ and let $\pi_{n}(C)$ be a point in $T$ such that

$$
e_{n, \pi_{n}(C)}^{\tau} \leqslant(1+\varepsilon) \inf \left\{e_{n, t}^{\tau}: t \in B\right\} .
$$

Consequently, for any $n \geqslant 2$ there exists a partition $\mathcal{B}_{C, n-2}$ of the set $U$ into at most $N_{n-2}$ sets $B$ that satisfy

$$
\Delta_{n+\tau-2, \pi_{n-2}(C)}(B) \leqslant 2 e_{n-2, \pi_{n-2}(C)}^{\tau}, \text { where } \Delta_{n, t}(B)=\sup _{u, v \in B} q_{n, t}(u, v) .
$$

Using sets $B \times C$ for $B \in \mathcal{B}_{C, n-2}$ and $C \in \mathcal{C}_{n-2}$ we get a partition $\mathcal{A}_{n-2}^{\prime}$ of $U \times T$ into at most $N_{n-2}^{2} \leqslant N_{n-1}$ sets. Finally intersecting all the constructed sets in $\mathcal{A}_{0}^{\prime}, \mathcal{A}_{1}^{\prime}, \ldots, \mathcal{A}_{n-2}^{\prime}$ we obtain a nested sequence of partitions $\left(\mathcal{A}_{n}\right)_{n \geqslant 2}$ such that $\left|\mathcal{A}_{n}\right| \leqslant N_{n}$. We complete the sequence by $\mathcal{C}_{0}=\mathcal{C}_{1}=\{U \times T\}$. In this way $\mathcal{A}=\left(\mathcal{A}_{n}\right)_{n \geqslant 0}$ is an admissible sequence of partitions for $U \times T$. Let $A_{n}(u, t)$ be the element of $\mathcal{A}_{n}$ that contains point $(u, t)$. Clearly

$$
A_{n}(u, t) \subset B \times C,
$$

where $C=C_{n-2}(t)$ and $u \in B \in \mathcal{B}_{C, n-2}$. Therefore for $n \geqslant 2$,

$$
\sup _{s, s^{\prime} \in C} q_{n+\tau-2, U}\left(s, s^{\prime}\right) \leqslant \Delta_{n+\tau-2, U}(C)
$$

and

$$
\sup _{v, v^{\prime} \in B} q_{n+\tau-2, \pi_{n}(C)}\left(v, v^{\prime}\right) \leqslant 2 e_{n-2, \pi_{n-2}(C)}^{\tau} \leqslant 2(1+\varepsilon) e_{n-2, t}^{\tau} .
$$

We turn to the analysis of optimal quantiles $\bar{q}_{n}$ for the process $X(u, t), u \in \mathcal{A}$, $t \in T$. We show that for any $x, y, z \in T$ and $v, w \in U$

$$
\bar{q}_{n}((v, x),(w, y)) \leqslant q_{n, U}(x, z)+q_{n, U}(y, z)+q_{n, z}(v, w) .
$$


This holds due to the triangle inequality

$$
\begin{aligned}
& \bar{q}_{n}((v, x),(w, y)) \\
& \leqslant \bar{q}_{n}((v, x),(v, z))+\bar{q}_{n}((w, y),(w, z))+\bar{q}_{n}((v, z),(w, z)) \\
& \leqslant q_{n, v}(x, z)+q_{n, w}(y, z)+q_{n, z}(v, w) \\
& \leqslant q_{n, U}(x, z)+q_{n, U}(y, z)+q_{n, z}(v, w) .
\end{aligned}
$$

In particular it implies that for any $(v, s) \in B \times C$

$$
\begin{aligned}
& \bar{q}_{n+\tau-2}((u, t),(v, s)) \\
& \leqslant q_{n+\tau-2, U}\left(t, \pi_{n-2}(C)\right)+q_{n+\tau-2, U}\left(s, \pi_{n-2}(C)\right)+q_{n+\tau-2, \pi_{n-2}(C)}(u, v)
\end{aligned}
$$

and hence

$$
\Delta_{n+\tau-2}(B \times C) \leqslant 2 \Delta_{n+\tau-2, U}\left(C_{n-2}(t)\right)+2(1+\varepsilon) e_{n-2, t}^{\tau} .
$$

If $\tau \geqslant 2$ then also for $n=0,1$,

$$
\Delta_{n+\tau-2}(U \times T) \leqslant \Delta_{\tau}(U \times T) \leqslant 2 \Delta_{\tau}(T)+2(1+\varepsilon) \sup _{t \in T} e_{0, t}^{\tau} .
$$

It implies that for any $\tau \geqslant 2$

$\gamma_{X}^{\tau-2}(U \times T) \leqslant 4 \Delta_{\tau}(T)+4(1+\varepsilon) \sup _{t \in T} e_{0, t}^{\tau}+2(1+\varepsilon) \gamma_{X, U}^{\tau}(T)+2(1+\varepsilon) \mathcal{E}_{X, T}^{\tau}(U)$.

Therefore for any $\tau \geqslant 4$ we may apply Theorem 1.3 for distances $\bar{q}_{n}$ and in this way prove our result with the constant 24 .

In the next sections we analyse various applications of the result.

\section{Gaussian case}

As we have mentioned the basic example for the theory are Gaussian canonical processes. Suppose that $T \subset \ell^{2}(I)$, we recall that

$$
G(t)=\sum_{i \in I} t_{i} g_{i}, \quad t \in T,
$$

where $\left(g_{i}\right)_{i \in I}$ is a sequence of i.i.d. standard normal variables. For the process $G(t), t \in T$ the natural distance is

$$
d(s, t)=\left(\mathbf{E}|G(t)-G(s)|^{2}\right)^{\frac{1}{2}}=\|t-s\|_{2}, \quad s, t \in T .
$$

It is easy to see that the optimal quantiles for $G$ satisfy

$$
\bar{q}_{n}(s, t) \sim 2^{\frac{n}{2}} d(s, t), \text { for all } s, t \in T .
$$

Consequently denoting $q_{n}(s, t)=C 2^{\frac{n}{2}} d(s, t)$ for large enough $C$ we get an admissible sequence of distances. Moreover,

$$
\gamma_{G}(T) \sim \gamma_{2}(T, d)=\inf _{\mathcal{A}} \sup _{t \in T} \sum_{n=0}^{\infty} 2^{\frac{n}{2}} \Delta\left(A_{n}(t)\right)
$$


where the infimum is taken over all admissible $\mathcal{A}=\left(\mathcal{A}_{n}\right)_{n \geqslant 0}$ sequences of partitions and $\Delta(A)=\sup _{s, t \in A} d(s, t)$. Since $d$ is the canonical distance for $\ell^{2}(I)$ we usually suppress $d$ from $\gamma_{2}(T, d)$ and simply write $\gamma_{2}(T)$. As we have mentioned in the introduction, using (1.3) we get

$$
K^{-1} \gamma_{2}(T) \leqslant \mathbf{E} \sup _{s, t \in T}|G(t)-G(s)| \leqslant K \gamma_{2}(T) .
$$

Let us also define

$$
e_{n}=\inf \left\{\varepsilon: N(T, d, \varepsilon) \leqslant N_{n}\right\}, n \geqslant 0 .
$$

Obviously $e_{n}^{\tau} \leqslant C 2^{\frac{n+\tau}{2}} e_{n}$ and hence

$$
\mathcal{E}_{G}^{\tau}(T) \leqslant C 2^{\frac{\tau}{2}} \sum_{n \geqslant 0} 2^{\frac{n}{2}} e_{n} .
$$

Let $\mathcal{E}(T, d)=\sum_{n=0}^{\infty} 2^{\frac{n}{2}} e_{n}$, it is the well known fact (Theorem 3.1.1 in [15]) that

$$
K^{-1} \mathcal{E}(T, d) \leqslant \int_{0}^{\infty} \sqrt{\log (N(T, d, \varepsilon))} d \varepsilon \leqslant K \mathcal{E}(T, d) .
$$

Again since $d$ is the canonical distance on $\ell^{2}(I)$ we will suppress $d$ from $\mathcal{E}(T, d)$ and write $\mathcal{E}(T)$. By (2.3) we infer Dudley's bound

$$
\mathbf{E} \sup _{s, t \in T}|G(t)-G(s)| \leqslant 16 C 2^{\frac{\tau}{2}} \mathcal{E}(T) .
$$

Turning our attention to product spaces let us recall that for $U \subset \mathbb{R}^{I}$ or $\mathbb{C}^{I}$, $T \subset \mathbb{R}^{I}$ or $C^{I}$ such that $u t=\left(u_{i} t_{i}\right)_{i \in I} \in \ell^{2}(I)$ for all $u \in U$ and $t \in T$ we may define

$$
G(u, t)=\sum_{i \in I} u_{i} t_{i} g_{i}, \quad u \in U, t \in T,
$$

where $\left(g_{i}\right)_{i \in I}$ is a Gaussian sequence. Note that for all $s, t \in T, u, v \in U$

$$
\bar{q}_{n}((u, t),(v, s)) \leqslant C 2^{\frac{n}{2}}\|u t-v s\|_{2} .
$$

For each $u \in U$ and $s, t \in T$ let $d_{u}(s, t)=\|u(t-s)\|_{2}$. We may define

$$
q_{n, u}(s, t)=C 2^{\frac{n}{2}} d_{u}(s, t), \quad q_{n, U}(s, t)=C 2^{\frac{n}{2}} \sup _{u \in U} d_{u}(s, t) .
$$

In particular

$$
q_{n, U}(s, t) \leqslant C \sup _{u \in U}\|u\|_{\infty} d(s, t)
$$

and therefore

$$
\gamma_{G, U}^{\tau}(T) \leqslant C 2^{\frac{\tau}{2}} \sup _{u \in U}\|u\|_{\infty} \gamma_{2}(T, d) .
$$

On the other hand we define for all $t \in T$ and $u, v \in U$

$$
q_{n, t}(u, v)=C 2^{\frac{n}{2}}\|t(u-v)\|_{2} .
$$

For each $t \in T$ let us denote by $d_{t}$ the distance on $U$ given by

$$
d_{t}(u, v)=\left(\sum_{i \in I}\left|t_{i}\right|^{2}\left|u_{i}-v_{i}\right|^{2}\right)^{\frac{1}{2}}, u, v \in U
$$


Using these distances we may rewrite (3.4) as $q_{n, t}=C 2^{\frac{n}{2}} d_{t}$. Let

$$
e_{n, t}=\inf \left\{\varepsilon: N\left(U, d_{t}, \varepsilon\right) \leqslant N_{n}\right\}, n \geqslant 0 \text { and } \mathcal{E}\left(U, d_{t}\right)=\sum_{n=0}^{\infty} 2^{\frac{n}{2}} e_{n, t},
$$

we obtain by (3.4)

$$
e_{n, t}^{\tau} \leqslant C 2^{\frac{n+\tau}{2}} e_{n, t} \text { and } \mathcal{E}_{X, T}^{\tau}(U) \leqslant C 2^{\frac{\tau}{2}} \sup _{t \in T} \mathcal{E}\left(U, d_{t}\right) .
$$

Using (3.2) we have

$$
K^{-1} \mathcal{E}\left(U, d_{t}\right) \leqslant \int_{0}^{\infty} \sqrt{\log \left(N\left(U, d_{t}, \varepsilon\right)\right)} d \varepsilon \leqslant K \mathcal{E}\left(U, d_{t}\right) .
$$

We recall also that if $0 \in T$ then by (1.3) $\gamma_{2}(T) \sim g(T)=\mathbf{E} \sup _{t \in T}|G(t)|$. We may state the following corollary of Theorem 2.3 which extends slightly Theorem 3.3.1 in [15].

Corollary 3.1 For any $\tau \geqslant 4$

$$
\begin{aligned}
& \mathbf{E} \sup _{u, v \in U} \sup _{s, t \in T}|G(u, t)-G(v, s)| \\
& \leqslant 32\left(\gamma_{G, U}^{\tau}(T)+\mathcal{E}_{G, T}^{\tau}(U)\right) \leqslant 32 C 2^{\frac{\tau}{2}}\left(\sup _{u \in U}\|u\|_{\infty} \gamma_{2}(T)+\sup _{t \in T} \mathcal{E}\left(U, d_{t}\right)\right) .
\end{aligned}
$$

Moreover

$$
\mathcal{E}\left(U, d_{t}\right) \sim \int_{0}^{\infty} \sqrt{\log N\left(U, d_{t}, \varepsilon\right)} d \varepsilon \text { and } \gamma_{2}(T) \sim g(T) \text { if } 0 \in T .
$$

It is a tempting idea to replace $\sup _{u \in U}\|u\|_{\infty} \gamma_{2}(T)$ and $\sup _{t \in T} \mathcal{E}\left(U, d_{t}\right)$ by respectively $\sup _{u \in U} \gamma_{2}\left(T, d_{u}\right)$ and $\sup _{t \in T} \gamma_{2}\left(U, d_{t}\right)$. We show that this approach cannot work, to this aim lets us consider toy example where $T$ and $U$ are usual ellipsoids, i.e.

$$
U=\left\{u \in \mathbb{R}^{I}: \sum_{i \in I} \frac{\left|u_{i}\right|^{2}}{\left|x_{i}\right|^{2}} \leqslant 1\right\} .
$$

and

$$
T=\left\{t \in \mathbb{R}^{I}: \sum_{i \in I} \frac{\left|t_{i}\right|^{2}}{\left|y_{i}\right|^{2}} \leqslant 1\right\} .
$$

Obviously

$$
\mathbf{E} \sup _{u \in U, t \in T}|G(u, t)|=\mathbf{E} \max _{i \in I}\left|x_{i} y_{i} g_{i}\right|
$$

on the other hand

$$
\sup _{u \in U} \mathbf{E} \sup _{t \in T}|G(u, t)| \sim \sup _{u \in U}\|u y\|_{2}=\max _{i \in I}\left|x_{i} y_{i}\right|=\|x y\|_{\infty} .
$$

Similarly, $\sup _{t \in T} \mathbf{E} \sup _{u \in U}|G(u, t)| \sim\|x y\|_{\infty}$. However $\|x y\|_{\infty} \leqslant 1$ does not guarantee that $\mathbf{E} \max _{i \in I}\left|x_{i} y_{i} g_{i}\right|$ is finite, for example if $x_{i}=y_{i}=1$. On the other hand Corollary 3.1 implies the following result for the ellipsoid $U$. 
Remark 3.2 Suppose that $\mathcal{A}$ is given by (3.5). Then for any set $0 \in T \subset \ell^{2}(I)$,

$$
\mathbf{E} \sup _{u \in U} \sup _{t \in T}|G(u, t)| \leqslant K\left(\|x\|_{\infty} g(T)+\Delta(T)\|x\|_{2}\right) .
$$

Proof. For the sake of simplicity we assume that $I=\mathbb{N}$. We use Corollary 3.1 together with the following observation. For each $t \in T$ points $\left(u_{i} t_{i}\right)_{i \in \mathbb{N}}$, $u \in U$ forms an ellipsoid $U_{t}=\left\{a \in \mathbb{R}^{\mathbb{N}}: \sum_{i \in \mathbb{N}} \frac{\left|u_{i}\right|^{2}}{\left|t_{i}\right|^{2}\left|x_{i}\right|^{2}} \leqslant 1\right\}$ and therefore by Proposition 2.5.2 in [15]

$$
\int_{0}^{\infty} \sqrt{\log N\left(U, d_{t}, \varepsilon\right)} d \varepsilon \leqslant L \sum_{n=0}^{\infty} 2^{\frac{n}{2}}\left|x_{2^{n}} t_{2^{n}}\right|,
$$

where $L$ is a universal constant, assuming that we have rearranged $\left(x_{i} t_{i}\right)_{i \in \mathbb{N}}$ in such a way that the sequence $\left|x_{i} t_{i}\right|, i \in \mathbb{N}$ is non-increasing. It suffices to note that by the Schwartz inequality

$$
\begin{aligned}
& \sum_{n=0}^{\infty} 2^{\frac{n}{2}}\left(\left|x_{2^{n}} t_{2^{n}}\right|^{2}\right) \leqslant 2\left|x_{1} t_{1}\right|+2 \sum_{n=1}^{\infty}\left(\sum_{i=2^{n-1}+1}^{2^{n}}\left|x_{i} t_{i}\right|^{2}\right)^{\frac{1}{2}} \\
& \leqslant 2\left|x_{1} t_{1}\right|+2 \sum_{n=1}^{\infty} \max _{2^{n-1}<i \leqslant 2^{n}}\left|x_{i}\right|\left(\sum_{i=2^{n-1}+1}^{2^{n}}\left|t_{i}\right|^{2}\right)^{\frac{1}{2}} \leqslant 2\|x\|_{2}\|t\|_{2} .
\end{aligned}
$$

Consequently,

$$
\mathbf{E} \sup _{u \in U} \sup _{t \in T}\left|G_{t, u}\right| \leqslant K\left(\|x\|_{\infty} \gamma_{2}(T)+\Delta(T)\|x\|_{2}\right) .
$$

It remains to apply (1.3), i.e. $g(T) \sim \gamma_{2}(T)$.

Note that $\|x\|_{\infty}=\sup _{u \in U}\|u\|_{\infty}$ and $\|x\|_{2} \sim \gamma_{2}(U, d)$. It is clear the result can be slightly improved if $\mathcal{E}(U, d)<\infty$. Indeed similarly to (3.3) one can show that

$$
\mathcal{E}_{G, U}^{\tau}(T) \leqslant C 2^{\frac{\tau}{2}} \sup _{t \in T}\|t\|_{\infty} \mathcal{E}(U, d)
$$

and hence by Corollary 3.1 for any set $U$ such that $\mathcal{E}(U, d)<\infty$

$$
\mathbf{E} \sup _{u \in U} \sup _{t \in T}|G(u, t)| \leqslant K\left(\|x\|_{\infty} g(T)+\sup _{t \in T}\|t\|_{\infty}\right) .
$$

This is a sort of general rule that we expect certain regularity of $\mathcal{A}$, whereas set $T$ is usually supposed to be unknown.

We turn to show that Corollary 3.1 is sufficiently strong to answer both questions about VC classes and Fourier series, posed in the introduction, in the Gaussian type formulation. Namely we prove that these problems are related to certain properties of entropy functionals $\int_{0}^{\infty} \sqrt{\log \left(N\left(\mathcal{A}, d_{t}, \varepsilon\right)\right)} d \varepsilon, t \in T$.

We start from the result on $\mathrm{VC}$ classes of finite dimension. In this case $\mathcal{A}$ consists of $a \in \mathbb{R}^{I}$, of the form $a=1_{A}$ for some $A \subset I$ and $T$ is any subset of 
$\ell^{2}(I)$ that contains 0. By Theorem 14.12 in [1] we have that for any $t \in \ell_{2}(I)$ such that $\|t\|_{2}=1$ and given $\mathrm{VC}$ class $\mathcal{A}$ of dimension $d$

$$
\log N\left(\mathcal{A}, d_{t}, \varepsilon\right) \leqslant L d\left(1+\log \frac{1}{\varepsilon}\right), \quad 0<\varepsilon<1 .
$$

Consequently

$$
\sup _{t \in T} \int_{0}^{\infty} \sqrt{\log N\left(\mathcal{A}, d_{t}, \varepsilon\right)} d \varepsilon \leqslant \sqrt{L d} \Delta(T) \int_{0}^{1}\left(1+\log \frac{1}{\varepsilon}\right)^{\frac{1}{2}} d \varepsilon \leqslant M \sqrt{d} \Delta(T)
$$

and hence $\mathcal{E}\left(\mathcal{A}, d_{t}\right) \leqslant M \sqrt{d} \Delta(T)$ for any $t \in T$. Since clearly $\Delta(T) \leqslant \gamma_{2}(T)$ we may use Corollary 3.1 for $\tau=4$ and deduce

$$
\mathbf{E} \sup _{a \in \mathcal{A} \sup _{t \in T}}|G(a, t)| \leqslant 64 C\left(\gamma_{2}(T)+M \sqrt{d} \Delta(T)\right) \leqslant L \sqrt{d} \gamma_{2}(T),
$$

which by (1.3) implies (1.5) - the result we refer to.

Next step is to show that the Gaussian version of the problem on Fourier series is also related to Corollary 3.1. In this case $U$ consists of $u \in \mathbb{C}^{I}$ of the form $u_{i}=$ $\chi_{i}(h)$ for $h \in G$, where $\chi_{i}, i \in I$ are characters on the compact Abelian group $G$ and $T$ is any subset of $\ell^{2}(I)$ that contains 0 . Recall that the crucial observation for the study is that distances $d_{t}, t \in T$ defined in (1.12) are translationally invariant on the group $G$, i.e.

$$
d_{t}(f \cdot h, g \cdot h)=d_{t}(f, g)=\left(\sum_{i \in I}\left|t_{i}\right|^{2}\left|\chi_{i}(f)-\chi_{i}(g)\right|^{2}\right)^{\frac{1}{2}}, \text { for any } f, g, h \in G .
$$

Therefore by the deep result of Fernique (Theorem 3.1.1 in [15]) we have

$$
K^{-1} \mathcal{E}\left(\mathcal{A}, d_{t}\right) \leqslant \mathbf{E} \sup _{a \in \mathcal{A}}|G(a, t)| \leqslant K \mathcal{E}\left(\mathcal{A}, d_{t}\right)
$$

Consequently by Corollary 3.1 and (1.3) we can deduce (1.7) which is the Gaussian version of Theorem 1.3 .

The first new consequence of Corollary 3.1 concerns shattering dimension of $U$. Suppose that $U$ is the class of real functions bounded by 1 , i.e. $U \subset[-1,1]^{I}$. We say that a subset $B$ of $I$ is $\varepsilon$-shattered if there exists a level function $v$ on $B$ such that given any subset $A$ of $B$ one can find a function $u \in U$ with $u_{i} \leqslant v_{i}$ if $i \in A$ and $u_{i} \geqslant v_{i}+\varepsilon$ if $i \in B \backslash A$. The shattering dimension of $U$ denoted by $\operatorname{vc}(U, \varepsilon)$ is the maximal cardinality of a set $\varepsilon$-shattered by $U$. The deep result of Mendelson and Vershynin [14] is that for any $t \in \ell_{2}(I)$ such that $\|t\|_{2}=1$ we have

$$
\log N\left(U, d_{t}, \varepsilon\right) \leqslant L \mathrm{vc}(U, c \varepsilon) \log \left(\frac{2}{\varepsilon}\right), \quad 0<\varepsilon<1,
$$

where $L$ and $c$ are positive absolute constants. This leads to the following corollary.

Corollary 3.3 Suppose that $0 \in T \subset \ell^{2}(I)$ and $\sup _{u \in U}\|u\|_{\infty}$. The following inequality holds

$$
\mathbf{E} \sup _{u \in U} \sup _{t \in T}|G(u, t)| \leqslant K\left(g(T)+\Delta(T) \int_{0}^{1} \sqrt{\operatorname{vc}(U, \varepsilon) \log (2 / \varepsilon)} d \varepsilon\right) .
$$


Proof. It suffices to apply (3.6) then Corollary 3.1 and finally (1.3).

It is worth mentioning how to understand the shattering dimension of convex bodies. Suppose that $U \subset[-1,1]^{d}$ is a convex and symmetric body then $\operatorname{vc}(U, \varepsilon)$ is the maximal cardinality of a subset $J$ of $\{1,2, \ldots, d\}$ such that $P_{J}(U) \supset$ $\left[-\frac{\varepsilon}{2}, \frac{\varepsilon}{2}\right]^{J}$, where $P_{J}$ is the orthogonal projection from $\mathbb{R}^{d}$ on $\mathbb{R}^{J}$. For example suppose that $U$ is a unit ball in $\mathbb{R}^{d}$ then $\operatorname{vc}(U, \varepsilon)=k-1$ for any $\varepsilon \in\left[\frac{2}{\sqrt{k}}, \frac{2}{\sqrt{k-1}}\right)$ and $k=1,2, \ldots d$ moreover $\operatorname{vc}(U, \varepsilon)=d$ for $\varepsilon<\frac{2}{\sqrt{d}}$. Consequently

$$
\int_{0}^{1} \sqrt{\operatorname{vc}(U, \varepsilon) \log (2 / \varepsilon)} d \varepsilon \leqslant K \sqrt{d \log d} .
$$

Note that for $t \in \mathbb{R}^{d}, t_{i}=1 / \sqrt{d}$ we have that $\int_{0}^{\infty} \sqrt{\log N\left(U, d_{t}, \varepsilon\right)} d \varepsilon$ is comparable to $\sqrt{d}$ and hence the above estimate is not far from this answer.

\section{Bernoulli case}

Our next aim is to obtain a version of Corollary 3.1 in the setting of Bernoulli processes. We recall that by Bernoulli processes we mean

$$
X(t)=\sum_{i \in I} t_{i} \varepsilon_{i}, \text { for } t \in T \subset \ell^{2}(I),
$$

where $\left(\varepsilon_{i}\right)_{i \in I}$ is a Bernoulli sequence. Moreover we denoted

$$
b(T)=\mathbf{E} \sup _{t \in T}|X(t)|
$$

and if $0 \in T$ then by Theorem 1.1 we have a geometrical characterization of $b(T)$. We turn to the analysis of Bernoulli processes on product spaces, namely we consider

$$
X(u, t)=\sum_{i \in I} u_{i} t_{i} \varepsilon_{i}, \quad t \in T, u \in U
$$

where $T \subset \mathbb{R}^{I}$ or $\mathbb{C}^{I}, U \subset \mathbb{R}^{I}$ or $\mathbb{C}^{I}$ and $\left(\varepsilon_{i}\right)_{i \in I}$ is a Bernoulli sequence. Our approach is to use Theorem 1.1] in order to extend Corollary 3.1 to the case of random signs.

Theorem 4.1 Suppose that $0 \in T$. Then there exists $\pi: T \rightarrow \ell^{2}$ such that $\|\pi(t)\|_{1} \leqslant L b(T)$ for all $t \in T, \pi(0)=0$ and

$$
\mathbf{E} \sup _{u \in U} \sup _{t \in T}|X(u, t)| \leqslant K\left(\sup _{u \in U}\|u\|_{\infty} b(T)+\sup _{t \in T} \mathcal{E}\left(U, d_{t-\pi(t)}\right)\right),
$$

where

$$
\mathcal{E}\left(U, d_{t-\pi(t)}\right) \sim \int_{0}^{\infty} \sqrt{\log N\left(U, d_{t-\pi(t)}, \varepsilon\right)} d \varepsilon .
$$

Proof. Obviously we may assume that $b(T)=\mathbf{E} \sup _{t \in T}\left|\sum_{i \in I} t_{i} \varepsilon_{i}\right|<\infty$. Therefore by Theorem 1.1 there exists a decomposition $T \subset T_{1}+T_{2}, T_{1}, T_{2} \subset$ $\ell_{2}(I)$ which satisfies $0 \in T_{2}$ and

$$
\max \left\{\sup _{t \in T_{1}}\|t\|_{1}, g\left(T_{2}\right)\right\} \leqslant L b(T),
$$


where $L$ is a universal constant. Hence combining Corollary 3.1 with (4.1)

$$
\begin{aligned}
& \mathbf{E} \sup _{u \in U} \sup _{t \in T}|X(u, t)| \\
& \leqslant C\left(\mathbf{E} \sup _{u \in U} \sup _{t \in T_{1}}|X(u, t)|+\mathbf{E} \sup _{u \in U} \sup _{t \in T_{2}}|X(u, t)|\right) \\
& \leqslant C\left(\sup _{u \in U}\|u\|_{\infty} \sup _{t \in T_{1}}\|t\|_{1}+\sqrt{\frac{\pi}{2}} \mathbf{E} \sup _{u \in U} \sup _{t \in T_{2}}|G(u, t)|\right) \\
& \leqslant C L\left(\sup _{u \in U}\|u\|_{\infty} b(T)+\sup _{t \in T_{2}} \int_{0}^{\infty} \sqrt{\log N\left(U, d_{t}, \varepsilon\right)} d \varepsilon\right) .
\end{aligned}
$$

The decomposition of $T$ into $T_{1}+T_{2}$ can be defined in a way that $T_{1}=\{\pi(t)$ : $t \in T\}$ and $T_{2}=\{t-\pi(t): t \in T\}$ where $\pi: T \rightarrow \ell_{2}$ is such that $\pi(0)=0$, $\|\pi(t)\|_{1} \leqslant L b(T)$ and $\gamma_{2}\left(T_{2}\right) \leqslant L b(T)$. It completes the proof.

Our remarks on the entropy function from the previous chapter shows that Theorem 4.1 solves both our questions from the introduction. In fact we can easily extend our result for the functional shattering dimension.

Corollary 4.2 Suppose that $\sup _{u \in U}\|u\|_{\infty} \leqslant 1$ and $U$ is of $\varepsilon$-shattering dimension $\operatorname{vc}(\mathcal{A}, \varepsilon)$ for $0<\varepsilon<1$, then

$$
\mathbf{E} \sup _{u \in U} \sup _{t \in T}|X(u, t)| \leqslant K b(T) \int_{0}^{1} \sqrt{\operatorname{vc}(U, \varepsilon) \log (2 / \varepsilon)} d \varepsilon .
$$

Proof. Obviously by Theorem 4.1

$$
\begin{aligned}
& \mathbf{E} \sup _{u \in U} \sup _{t \in T}|X(u, t)| \\
& \leqslant K\left(\sup _{u \in U}\|u\|_{\infty} b(T)+\sup _{t \in T}\|t-\pi(t)\|_{2} \int_{0}^{1} \sqrt{\operatorname{vc}(U, \varepsilon) \log (2 / \varepsilon)} d \varepsilon\right),
\end{aligned}
$$

where $\|\pi(t)\|_{1} \leqslant L b(T)$. Since

$$
\sup _{t \in T}\|t-\pi(t)\|_{2} \leqslant L b(T) \text { and } \sup _{u \in U}\|u\|_{\infty} \leqslant 1
$$

it implies that

$$
\mathbf{E} \sup _{u \in U} \sup _{t \in T}|X(u, t)| \leqslant K b(T) \int_{0}^{1} \sqrt{\operatorname{vc}(U, \varepsilon) \log (2 / \varepsilon)} d \varepsilon .
$$

Corollary 4.3 Suppose that $\sup _{u \in U}\|u\|_{\infty} \leqslant 1$ and $v_{i} \in F, i \in I$, then

$$
\mathbf{E} \sup _{u \in U}\left\|\sum_{i \in I} u_{i} v_{i} \varepsilon_{i}\right\| \leqslant K \mathbf{E}\left\|\sum_{i \in I} v_{i} \varepsilon_{i}\right\| \int_{0}^{1} \sqrt{\mathrm{vc}(U, \varepsilon) \log (2 / \varepsilon)} d \varepsilon .
$$

Note that in the same way as for the maximal inequality we may ask what is the right characterization of $U \subset \mathbb{R}^{I}, \sup _{u \in U}\|u\|_{\infty} \leqslant 1$ for which the inequality

$$
\mathbf{E} \sup _{u \in U}\left\|\sum_{i \in I} u_{i} X_{i}\right\| \leqslant K\left\|\sum_{i \in I} X_{i}\right\|,
$$


holds for any sequence of independent symmetric r.v.'s $X_{i}, i \in I$ which take values in a separable Banach space $F$. With the same proof as the first part of Theorem 1.2 one can show that $U$ should satisfy the condition

$$
\sup _{0<\varepsilon<1} \varepsilon \sqrt{\operatorname{vc}(U, \varepsilon)}<\infty .
$$

On the other hand Corollary 4.3 implies that

$$
\int_{0}^{1} \sqrt{\mathrm{vc}(U, \varepsilon) \log (2 / \varepsilon)} d \varepsilon<\infty
$$

is a sufficient condition for the inequality (4.2). The exact answer for this question seems to stay at the moment beyond our reach.

We revisit our toy example where $U$ is the ellipsoid

$$
U=\left\{u \in \mathbb{R}^{I}: \sum_{i \in I} \frac{\left|u_{i}\right|^{2}}{\left|x_{i}\right|^{2}} \leqslant 1\right\}
$$

where $\left|x_{i}\right|>0$ are given numbers. One can show the following result.

Remark 4.4 Suppose that $U$ is the usual ellipsoid. Then for any set $0 \in T \subset$ $\ell^{2}(I)$

$$
\mathbf{E} \sup _{u \in U} \sup _{t \in T}|X(u, t)| \leqslant K\|x\|_{2} b(T) .
$$

Proof. We may argue in a similar way as in Remark 3.2 using this time Theorem 4.1 and obtain

$$
\mathbf{E} \sup _{u \in U} \sup _{t \in T}|X(u, t)| \leqslant K\left(\|x\|_{\infty} b(T)+\sup _{t \in T}\|t-\pi(t)\|_{2}\|x\|_{2}\right) .
$$

Since $\Delta(T) \leqslant L b(T)$ and $\|\pi(t)\|_{1} \leqslant L b(T)$ we get $\sup _{t \in T}\|t-\pi(t)\|_{2} \leqslant L b(T)$ and therefore

$$
\mathbf{E} \sup _{u \in U} \sup _{t \in T}|X(u, t)| \leqslant K\|x\|_{2} b(T) .
$$

On the other hand by the Schwartz inequality

$$
\mathbf{E} \sup _{u \in U} \sup _{t \in T}|X(u, t)|=\sup _{t \in T} \mathbf{E} \sup _{u \in U}|X(u, t)|=\sup _{t \in T}\|t x\|_{2}
$$

Consequently in this case the expectation of the supremum of the Bernoulli process over the product of index sets can be explained by the analysis of one of its marginal processes.

It is worth mentioning that whenever there holds a comparability of moments like (4.2) one can deduce also the comparability of tails.

Remark 4.5 Suppose that $\sup _{u \in U}\|u\|_{\infty} \leqslant 1$. If for any $X_{i}, i \in I$ symmetric independent random variables which take values in a separable Banach space $(F,\|\cdot\|)$

$$
\mathbf{E} \sup _{u \in U}\left\|\sum_{i \in I} u_{i} X_{i}\right\| \leqslant L \mathbf{E}\left\|\sum_{i \in I} X_{i}\right\|,
$$


then there exists an absolute constant $K$ such that

$$
\mathbf{P}\left(\sup _{u \in U}\left\|\sum_{i \in I} u_{i} X_{i}\right\| \geqslant K t\right) \leqslant K \mathbf{P}\left(\left\|\sum_{i \in I} X_{i}\right\| \geqslant t\right) .
$$

Proof. It suffices to prove the inequality for $X_{i}=v_{i} \varepsilon_{i}$, where $v_{i} \in F$ and $\varepsilon_{i}$ are independent Bernoulli variables. The general result follows then from the Fubini theorem. By the result of Dilworth and Montgomery-Smith [2] for all $p \geqslant 1$

$$
\begin{aligned}
& \left\|\sup _{u \in U}\right\| \sum_{i \in I} u_{i} v_{i} \varepsilon_{i}\|\|_{p} \\
& \leqslant C\left(\left\|\sup _{u \in U}\right\| \sum_{i \in I} u_{i} v_{i} \varepsilon_{i}\|\|_{1}+\sup _{u \in U} \sup _{\left\|x^{*}\right\| \leqslant 1}\left\|\sum_{i \in I} u_{i} x_{i}^{*}\left(v_{i}\right) \varepsilon_{i}\right\|_{p}\right) .
\end{aligned}
$$

Therefore by the Markov inequality and the assumption $\sup _{u \in U}\|u\|_{\infty} \leqslant 1$ we obtain for all $p \geqslant 1$

$$
\begin{aligned}
& \mathbf{P}\left(\sup _{u \in U}\left\|\sum_{i \in I} u_{i} v_{i} \varepsilon_{i}\right\| \geqslant C\left(\mathbf{E}\left\|\sum_{i \in I} v_{i} \varepsilon_{i}\right\|+\sup _{\left\|x^{*}\right\| \leqslant 1}\left\|\sum_{i \in I} x^{*}\left(v_{i}\right) \varepsilon_{i}\right\|_{p}\right)\right) \\
& \leqslant e^{-p} .
\end{aligned}
$$

On the other hand it is known (e.g. [8]) that for any functional $x^{*} \in F^{*}$

$$
\mathbf{P}\left(\left|\sum_{i \in I} x^{*}\left(v_{i}\right) \varepsilon_{i}\right| \geqslant M^{-1}\left\|\sum_{i \in I} x^{*} v_{i} \varepsilon_{i}\right\|_{p}\right) \geqslant \min \left\{c, e^{-p}\right\},
$$

where $M \geqslant 1$. Hence

$$
\begin{aligned}
& \mathbf{P}\left(\sup _{u \in U}\left\|\sum_{i \in I} u_{i} v_{i} \varepsilon_{i}\right\| \geqslant C\left(\mathbf{E}\left\|\sum_{i \in I} v_{i} \varepsilon_{i}\right\|+M t\right)\right) \\
& \leqslant \sup _{\left\|x^{*}\right\| \leqslant 1} c^{-1} \mathbf{P}\left(\left|\sum_{i \in I} x^{*}\left(v_{i}\right) \varepsilon_{i}\right|>t\right) \leqslant c^{-1} \mathbf{P}\left(\left\|\sum_{i \in I} v_{i} \varepsilon_{i}\right\|>t\right) .
\end{aligned}
$$

We end the proof considering two cases. If $t \geqslant M^{-1} \mathbf{E}\left\|\sum_{i \in I} v_{i} \varepsilon_{i}\right\|$ then

$$
\mathbf{P}\left(\sup _{u \in U}\left\|\sum_{i \in I} u_{i} v_{i} \varepsilon_{i}\right\| \geqslant 2 C M t\right) \leqslant c^{-1} \mathbf{P}\left(\left\|\sum_{i \in I} v_{i} \varepsilon_{i}\right\|>t\right) .
$$

If $t \leqslant M^{-1} \mathbf{E}\left\|\sum_{i \in I} v_{i} \varepsilon_{i}\right\|$ then by Paley-Zygmund inequality and the Kahane inequality with the optimal constant [6]

$$
\mathbf{P}\left(\left\|\sum_{i \in I} v_{i} \varepsilon_{i}\right\|>t\right) \geqslant M^{-2} \frac{\left(\mathbf{E}\left\|\sum_{i \in I} \varepsilon_{i} v_{i}\right\|\right)^{2}}{\mathbf{E}\left\|\sum_{i \in I} \varepsilon_{i} v_{i}\right\|^{2}} \geqslant \frac{1}{2} M^{-2} .
$$


It shows that

$$
\mathbf{P}\left(\sup _{u \in U}\left\|\sum_{i \in I} u_{i} v_{i} \varepsilon_{i}\right\| \geqslant K t\right) \leqslant K \mathbf{P}\left(\left\|\sum_{i \in I} v_{i} \varepsilon_{i}\right\|>t\right),
$$

which completes the proof.

\section{Applications}

First application we show concerns processes on $\mathcal{A}=[0,1]$ but with values in $\mathbb{R}^{I}$. In order to discuss whether or not $X(a), a \in \mathcal{A}$ has its paths in $\ell^{2}(I)$ space we have to verify the condition $\|X(a)\|_{2}<\infty$ a.s. for all $a \in \mathcal{A}$. This is the same question as

$$
\sup _{\left\|x^{*}\right\| \leqslant 1} \sup _{a \in \mathcal{A}}\left\langle x^{*}, X(a)\right\rangle<\infty .
$$

Hence it suffices to check the condition $\mathbf{E s u p}_{a \in \mathcal{A}} \sup _{t \in T}|X(a, t)|<\infty$, where $T=\left\{t \in \ell^{2}(I):\|t\|_{2} \leqslant 1\right\}$ and

$$
X(a, t)=\langle t, X(a)\rangle, \quad t \in T, a \in \mathcal{A} \text {. }
$$

Note that in this way we match each random vector $X(a)$ with the process $X(a, t), t \in T$. By Theorem 2.3 (note that $X(0, s)=0$ )

$$
\mathbf{E} \sup _{a \in \mathcal{A}} \sup _{t \in T}|X(a, t)| \leqslant 32\left(\gamma_{X, \mathcal{A}}^{\tau}(T)+\mathcal{E}_{X, T}^{\tau}(\mathcal{A})\right) .
$$

In the described setting we usually expect that

$$
q_{n, a}(s, t) \leqslant q_{n, 1}(s, t), \quad a \in \mathcal{A}
$$

which means that on average the increments increases with time. Condition (5.1) yields $\gamma_{X, \mathcal{A}}^{\tau}(T) \leqslant \gamma_{X(1)}^{\tau}(T)$. Under certain assumptions we may also expect that $\gamma_{X(1)}^{\tau}(T)$ is equivalent to $\mathbf{E}\|X(1)\|_{2}$. For example this is the case when $X(1)$ is a centred Gaussian vector and also if $X(1)$ consists of entries $X(1)_{i}, i \in I$ that are independent random variables that satisfy some technical assumptions as stated in [10]. Moreover if there exists increasing family of functions $\eta_{n}$ : $\mathbb{R}_{+} \rightarrow \mathbb{R}_{+}$which are continuous, increasing, $\eta_{n}(0)=0$ such that for all $t \in T$ and $a, b \in \mathcal{A}$

$$
q_{n, t}(a, b) \leqslant \eta_{n}(|a-b|) \text { and } \sum_{n=0}^{\infty} \eta_{n+\tau}^{-1}\left(N_{n}\right)<\infty
$$

then

$$
\mathcal{E}_{X, T}^{\tau}(\mathcal{A}) \leqslant \sum_{n=0}^{\infty} \eta_{n+\tau}^{-1}\left(N_{n}\right)<\infty .
$$

In this way we obtain the following remark which is a generalization results from [3, 4. 
Remark 5.1 Suppose that $X(a, t), a \in \mathcal{A}, t \in T$ satisfies (5.1), (5.2) and $\mathbf{E}\|X(1)\|_{2}$ is comparable with $\gamma_{X(1)}(T)$ then

$$
\mathbf{E} \sup _{a \in \mathcal{A}} \sup _{t \in T}|X(a, t)| \leqslant K\left(\mathbf{E}\|X(1)\|_{2}+1\right) .
$$

The second application we would like to discuss concerns empirical processes. Let $(\mathcal{X}, \mathcal{B})$ be a measurable space, $\mathcal{F}$ be a countable family of measurable functions $f: \mathcal{X} \rightarrow \mathbb{R}$ such that $0 \in \mathcal{F}$ and $X_{1}, X_{2}, \ldots, X_{N}$ be family of independent random variables that satisfy

$$
\mathbf{P}\left(\left|(f-g)\left(X_{i}\right)\right|>d_{1}(f, g) t+d_{2}(f, g) t^{\frac{1}{2}}\right) \leqslant 2 \exp (-t), \text { for all } t>0 .
$$

We will analyse the case when $d_{2}(f, g) \geqslant d_{1}(f, g)$. By Exercise 9.3.5 in [15] for any centred independent random variables $Y_{1}, Y_{2}, \ldots, Y_{n}$ which satisfy

$$
\mathbf{P}\left(\left|Y_{i}\right| \geqslant A t+B t^{\frac{1}{2}}\right) \leqslant 2 \exp (-t) \text { for all } i=1,2, \ldots, N,
$$

where $A \leqslant B$ and for any numbers $u_{1}, u_{2}, \ldots, u_{N}$ we have

$$
\mathbf{P}\left(\left|\sum_{i=1}^{N} u_{i} Y_{i}\right| \geqslant L\left(A\|u\|_{\infty} t+B\|u\|_{2} t^{\frac{1}{2}}\right)\right) \leqslant 2 \exp (-t) \text { for all } t>1 .
$$

Observe that if we define

$$
X(u, f)=\frac{1}{\sqrt{N}} \sum_{i=1}^{N} \varepsilon_{i} u_{i} f\left(X_{i}\right), \quad u \in U, f \in \mathcal{F},
$$

where $U$ is a unit ball in $\mathbb{R}^{N}$ and $\left(\varepsilon_{i}\right)_{i=1}^{N}$ is a Bernoulli sequence independent of $X_{i}, i=1,2, \ldots, N$ then

$$
\mathbf{E} \sup _{u \in U} \sup _{f \in \mathcal{F}}|X(u, f)|=\mathbf{E} \sup _{f \in \mathcal{F}}\left(\frac{1}{N} \sum_{i=1}^{N}\left|f\left(X_{i}\right)\right|^{2}\right)^{\frac{1}{2}} .
$$

Clearly by (5.4) used to $Y_{i}=\varepsilon_{i}(f-g)\left(X_{i}\right)$ we get for all $t>1$

$$
\begin{aligned}
& \mathbf{P}\left(|X(u, f)-X(u, g)| \geqslant \frac{L}{\sqrt{N}}\left(d_{1}(f, g)\|u\|_{\infty} t+d_{2}(f, g)\|u\|_{2} t^{\frac{1}{2}}\right)\right) \\
& \leqslant 2 \exp (-t) .
\end{aligned}
$$

Thus in particular we can use

$$
q_{n, u}(f, g)=L\left(d_{1}(f, g)\|u\|_{\infty} 2^{n}+d_{2}(f, g)\|u\|_{2} 2^{\frac{n}{2}}\right) .
$$

Then

$$
q_{n, U}(f, g)=\frac{L}{\sqrt{N}}\left(d_{1}(f, g) 2^{n}+d_{2}(f, g) 2^{\frac{n}{2}}\right) .
$$

Let

$$
\gamma_{i}\left(\mathcal{F}, d_{i}\right)=\inf _{\mathcal{A}} \sup _{f \in \mathcal{F}} \sum_{n=0}^{\infty} 2^{\frac{n}{i}} \Delta_{i}\left(A_{n}(f)\right)
$$


where the infimum is taken over all admissible partitions $\mathcal{A}=\left(\mathcal{A}_{n}\right)_{n \geqslant 0}$ and $\Delta_{i}(A)=\sup _{f, g \in A} d_{i}(f, g)$. It is easy to construct an admissible partitions which works for both $\gamma_{1}\left(\mathcal{F}, d_{1}\right)$ and $\gamma_{2}\left(\mathcal{F}, d_{2}\right)$. Namely we consider partitions $\mathcal{A}^{1}=$ $\left(\mathcal{A}_{n}^{1}\right)_{n \geqslant 0}$ and $\mathcal{A}^{2}=\left(\mathcal{A}_{n}^{2}\right)_{n \geqslant 0}$ such that

$$
(1+\varepsilon) \gamma_{2}\left(\mathcal{F}, d_{i}\right) \geqslant \sup _{f \in \mathcal{F}} \sum_{n=0}^{\infty} 2^{\frac{n}{i}} \Delta_{i}\left(A_{n}^{i}(f)\right), \quad i=1,2,
$$

for some arbitrary small $\varepsilon>0$ and then define $\mathcal{A}=\left(\mathcal{A}_{n}\right)_{n \geqslant 0}$ by $\mathcal{A}_{n}=\mathcal{A}_{n-1}^{1} \cap$ $\mathcal{A}_{n-1}^{2}$ for $n \geqslant 1$ and $\mathcal{A}_{0}=\{\mathcal{F}\}$. Obviously $\mathcal{A}$ is admissible, moreover,

$$
\sup _{f \in \mathcal{F}} \sum_{n=0}^{\infty} 2^{\frac{n}{i}} \Delta_{i}\left(A_{n}(f)\right) \leqslant(1+\varepsilon) 2^{\frac{1}{i}} \gamma_{i}\left(\mathcal{F}, d_{i}\right), \quad i=1,2 .
$$

Using the partition $\mathcal{A}$ we derive from (5.6)

$$
\gamma_{X, \mathcal{A}}^{\tau}(\mathcal{F}) \leqslant \frac{2^{\tau+1}(1+\varepsilon)}{\sqrt{N}}\left(\gamma_{1}\left(\mathcal{F}, d_{1}\right)+\gamma_{2}\left(\mathcal{F}, d_{2}\right)\right)
$$

On the other hand using that $X(u, f)-X(v, f)=X(u-v, f)-X(0, f)$ similarly to (5.5) we get for all $t>1$

$$
\begin{aligned}
& \mathbf{P}\left(|X(u, f)-X(v, f)| \geqslant \frac{L}{\sqrt{N}}\left(d_{1}(f, 0)\|u-v\|_{\infty} t+d_{2}(f, 0)\|u-v\|_{2} t^{\frac{1}{2}}\right)\right) \\
& \leqslant 2 \exp (-t) .
\end{aligned}
$$

Hence we may define

$$
q_{n, f}(u, v)=\frac{L}{\sqrt{N}}\left(d_{1}(f, 0)\|u-v\|_{\infty} 2^{n}+d_{2}(f, 0)\|u-v\|_{2} 2^{\frac{n}{2}}\right) .
$$

We aim to compute entropy numbers $e_{n, f}, n \geqslant 0$. Let $n_{0} \geqslant 1$ be such that $2^{n_{0}} \geqslant N \geqslant 2^{n_{0}-1}$. Our first claim is that for any $n \geqslant n_{0}$ and suitably chosen $\sigma \geqslant 1$ it is possible to cover $B_{N}(0,1)$, a unit ball in $\mathbb{R}^{N}$, by at most $N_{n+\sigma}$ cubes of $\ell^{\infty}$ diameter at most $N^{-\frac{1}{2}}\left(N_{n}\right)^{-\frac{1}{N}}$. Indeed we can apply the volume type argument. It is possible to cover $B_{N}(0,1)$ with $M$ disjoint cubes of $\ell^{\infty}$ diameter $t$ with disjoint interiors in $B_{N}(0,1+t \sqrt{N})$. Since $\left|B_{N}(0,1)\right| \sim(C / \sqrt{N})^{N}$ we get

$$
M \leqslant \frac{\left|B_{N}(0,1+t \sqrt{N})\right|}{t^{N}} \leqslant\left(\frac{C}{\sqrt{N}}\left(\frac{1+t \sqrt{N}}{t}\right)\right)^{N} \leqslant C^{N}\left(1+\frac{1}{t \sqrt{N}}\right)^{N} .
$$

We may choose $t=N^{-\frac{1}{2}} N_{n}^{-\frac{1}{N}}$, then

$$
C^{N}\left(1+\frac{1}{t \sqrt{N}}\right)^{N}=C^{N}\left(1+N_{n}^{\frac{1}{N}}\right)^{N} \leqslant\left(N_{n+\sigma}\right)^{\frac{1}{N}},
$$

where the last inequality uses the assumption that $n \geqslant n_{0}$. In this way we covered $B_{N}(0,1)$ by at most $N_{n+\sigma}$ sets of $\ell_{\infty}$ diameter at most $N^{-\frac{1}{2}}\left(N_{n}\right)^{-\frac{1}{N}}$ and $\ell_{2}$ diameter at most $N_{n}^{-\frac{1}{N}}$. By (5.8) we infer the following entropy bound

$$
e_{n+\sigma, f}^{\tau} \leqslant L 2^{\tau}\left(d_{1}(f, 0) N^{-1}\left(N_{n}\right)^{-\frac{1}{N}}+d_{2}(f, 0) N^{-\frac{1}{2}}\left(N_{n}\right)^{-\frac{1}{N}}\right) .
$$


We recall that constant $L$ is absolute but may change its value from line to line up to a numerical factor. This implies the bound

$$
\begin{aligned}
& \sum_{n=n_{0}}^{\infty} e_{n+\sigma, f}^{\tau} \\
& \leqslant L 2^{\tau}\left(d_{1}(f, 0) N^{-1}\left(N_{n}\right)^{-\frac{1}{N}}+d_{2}(f, 0) N^{-\frac{1}{2}}\left(N_{n}\right)^{-\frac{1}{N}}\right) \\
& \leqslant L 2^{\tau}\left(d_{1}(f, 0)+d_{2}(f, 0)\right) .
\end{aligned}
$$

The second step is to consider $n \leqslant n_{0} / 2+\sigma$. In this case we can simply use the trivial covering of $B_{N}(0,1)$ by a single set which obviously has $\ell^{\infty}$ and $\ell^{2}$ diameter equal 2 and hence

$$
e_{n, f}^{\tau}=\frac{L 2^{\tau}}{\sqrt{N}}\left(d_{1}(f, 0) 2^{n}+d_{2}(f, 0) 2^{\frac{n}{2}}\right)
$$

and

$$
\sum_{n=0}^{n_{0} / 2+\sigma} e_{n, f}^{\tau} \leqslant L 2^{\tau}\left(d_{1}(f, 0)+d_{2}(f, 0)\right) .
$$

The most difficult case is when $n_{0} / 2+\sigma \leqslant n \leqslant n_{0}+\sigma$. In this setting we will cover $B_{N}(0,1)$ with cubes of $\ell^{\infty}$ diameter $2 t$, where on $t=\frac{1}{\sqrt{m_{n}}}$ and $m_{n} \leqslant N$. We will not control $\ell^{2}$ diameter, we simply use that it is always bounded by 2. Note that if $x \in B_{N}(0,1)$ then only on at most $m_{n}$ coordinates such that $\left|x_{i}\right|>t$. Therefore we can cover $B_{N}(0,1)$ with cubes in $\mathbb{R}^{N}$ of $\ell^{\infty}$ diameter $2 t$ if for each subset $J \subset\{1,2, \ldots, N\}$ such that $|J|=m_{n}$ we cover $B_{J}(0,1) \subset \mathbb{R}^{J}$ with cubes in $\mathbb{R}^{J}$ of $\ell^{\infty}$ diameter $2 t$. In this way we cover all the cases where all coordinates but those in $J$ stay in the cube $[-t, t]^{J^{c}}$. By our volume argument one needs at most

$$
M_{J} \leqslant C^{J}\left(1+\frac{2 t}{\sqrt{|J|}}\right)^{|J|}=C^{m_{n}}\left(1+\frac{1}{2 t \sqrt{m_{n}}}\right)^{m_{n}}
$$

$2 t$-cubes in $\mathbb{R}^{J}$ to cover $B_{J}(0,1)$. Our choice of $t$ guarantees that $M_{J} \leqslant$ $(3 c / 2)^{m_{n}}$. Therefore one needs $\left(\begin{array}{c}N \\ m_{n}\end{array}\right)(3 c / 2)^{m_{n}}$ cubes of $\ell^{\infty}$ diameter $2 t$ to cover $B_{N}(0,1)$. We require that

$$
\left(\begin{array}{c}
N \\
m_{n}
\end{array}\right)\left(\frac{3 c}{2}\right)^{m_{n}} \leqslant N_{n+\sigma}
$$

It remains to find $m_{n}$ that satisfies the above inequality. First observe that

$$
\left(\begin{array}{c}
N \\
m_{n}
\end{array}\right) \leqslant\left(\frac{e N}{m_{n}}\right)^{m_{n}}=\exp \left(m_{n} \log \left(e N / m_{n}\right)\right)
$$

Following Talagrand we define $m_{n}$ as the smallest integer such that

$$
2^{n-\sigma} \leqslant m_{n} \log \left(e N / m_{n}\right) .
$$

Clearly if $n \geqslant n_{0} / 2+\sigma$ then $m_{n}>1$ and thus by Lemma 9.3.12 in [15] we deduce that $m_{n} \log \left(e N / m_{n}\right) \leqslant 2^{n-\sigma+1}$ and hence

$$
\left(\begin{array}{c}
N \\
m_{n}
\end{array}\right)\left(\frac{3 c}{2}\right)^{m_{n}} \leqslant \exp \left(2^{n-\sigma+1}\right)\left(\frac{3 c}{2}\right)^{m_{n}} \leqslant N_{n+\sigma}
$$


for sufficiently large $\sigma$. Again by Lemma 9.3 .12 in [15] we have $\frac{1}{8} m_{n+1} \leqslant m_{n}$ for all $n \in\left\{n_{0} / 2+\sigma, \ldots n_{0}+\sigma-1\right\}$ and $m_{n_{0}+\sigma}=2^{n_{0}} \geqslant N$. It implies that

$$
m_{n} \geqslant N\left(\frac{1}{8}\right)^{n_{0}+\sigma-n} .
$$

Recall that each of the covering cubes has $\ell^{2}$ diameter 2 and therefore by the definition of $m_{n}$ and then by (5.9)

$$
\begin{aligned}
& e_{n, f}^{\tau} \leqslant \frac{L 2^{\tau}}{\sqrt{N}}\left(d_{1}(f, 0) \frac{2^{n}}{\sqrt{m_{n}}}+d_{2}(f, 0) 2^{\frac{n}{2}}\right) \\
& \leqslant L 2^{\tau+\frac{n-n_{0}}{2}}\left(d_{1}(f, 0) \sqrt{\log \left(e N / m_{n}\right)}+d_{2}(f, 0)\right) \\
& \leqslant L 2^{\tau+\frac{n-n_{0}}{2}}\left(d_{1}(f, 0) \sqrt{1+\left(n_{0}+\sigma-n\right) \log (8)}+d_{2}(f, 0)\right) .
\end{aligned}
$$

Again we derive the bound

$$
\sum_{n=n_{0} / 2+\sigma}^{n_{0}+\sigma} e_{n, f}^{\tau} \leqslant L 2^{\tau}\left(d_{1}(f, 0)+d_{2}(f, 0)\right) .
$$

We have established that

$$
\mathcal{E}_{X, f}^{\tau}(U)=\sum_{n=0}^{\infty} e_{n, f}^{\tau} \leqslant L 2^{\tau}\left(d_{1}(f, 0)+d_{2}(f, 0)\right)
$$

and consequently

$$
\mathcal{E}_{X, \mathcal{F}}^{\tau}(U)=\sup _{f \in \mathcal{F}} \mathcal{E}_{X, f}^{\tau}(U) \leqslant L 2^{\tau} \sup _{f \in \mathcal{F}}\left(d_{1}(f, 0)+d_{2}(f, 0)\right) .
$$

By Theorem 2.3 we get

$$
\begin{aligned}
& \mathbf{E} \sup _{f \in \mathcal{F}}\left(\frac{1}{\sqrt{N}} \sum_{i=1}^{N}\left|f\left(X_{i}\right)\right|^{2}\right)^{\frac{1}{2}} \\
& \leqslant K\left(\frac{1}{\sqrt{N}}\left(\gamma_{1}\left(\mathcal{F}, d_{1}\right)+\gamma_{2}\left(\mathcal{F}, d_{2}\right)\right)+\Delta_{1}(\mathcal{F})+\Delta_{2}(\mathcal{F})\right) .
\end{aligned}
$$

Note that our assumption that $d_{2}$ dominates $d_{1}$ is not necessary for the result. Clearly using $\bar{d}_{2}=\max \left(d_{1}, d_{2}\right)$ instead of $d_{2}$ we only have to observe that for our admissible partition $\mathcal{A}$ which works for $\gamma_{1}\left(\mathcal{F}, d_{1}\right)$ and $\gamma_{2}\left(\mathcal{F}, d_{2}\right)$ in the sense of (5.7) one can use the following inequality

$$
\sum_{n=0}^{\infty} 2^{\frac{n}{2}} \bar{\Delta}_{2}\left(A_{n}(f)\right) \leqslant \sum_{n=0}^{\infty} 2^{n} \Delta_{1}\left(A_{n}(f)\right)+\sum_{n=0}^{\infty} 2^{\frac{n}{2}} \Delta_{2}\left(A_{n}(f)\right),
$$

where $\bar{\Delta}_{2}(A)$ is the diameter of $A$ with respect to $\bar{d}_{2}$ distance. In the same way $\bar{\Delta}_{2}(\mathcal{F}) \leqslant \Delta_{1}(\mathcal{F})+\Delta_{2}(\mathcal{F})$. We have proved the following result.

Theorem 5.2 Suppose that $0 \in \mathcal{F}$ and $\mathcal{F}$ satisfies (5.3). Then

$$
\begin{aligned}
& \mathbf{E} \sup _{f \in \mathcal{F}} \frac{1}{\sqrt{N}}\left(\sum_{i=1}^{N}\left|f\left(X_{i}\right)\right|^{2}\right)^{\frac{1}{2}} \\
& \leqslant K\left(\frac{1}{\sqrt{N}}\left(\gamma_{1}\left(\mathcal{F}, d_{1}\right)+\gamma_{2}\left(\mathcal{F}, d_{2}\right)\right)+\Delta_{1}(\mathcal{F})+\Delta_{2}(\mathcal{F})\right) .
\end{aligned}
$$


The result is due to Mendelson and Paouris [13] (see Theorem 9.3.1 in [15]) and concerns slightly more general situation. The proof we have shown is different and much less technically involved.

\section{References}

[1] W. Bednorz and R. Latala (2014) On the boundedness of Bernoulli processes, Ann. Math., 180 1167-1203.

[2] S. Dilworth (1993) The distribution of vector-valued Rademacher series, Ann. Probab., 21, 2046-2052

[3] X. Fernique (1992) Sur la regularite des fonctions aleatoires D'Ornstein-Uhlenbeck valeurs dans $l_{p}, p \in[1, \infty)$, Ann. Probab., 20, 1441-1449.

[4] X. Fernique (1997) Fonctions aléatoires Gaussiesnes, vecteurs aléatoires Gaussienses, Université de Montréal, Centre de Recherchers Mathématiques, Montreal QC.

[5] L. Krawczyk (1996) Maximal inequality for Gaussian vectors, Bull. Acad. Polon. Sci. Math., 44, 157-160.

[6] R.Latala And K. OleszKiewicz (1994) On the best constant in the Khintchin-Kahane inequality. Studia. Math., 109, 101-104.

[7] R. Latala (1999) A note on the maximal inequality for VC-classes, Advances in stochastic inequalities (Atlanta, GA, 1997), 125-134, Contemp. Math. 234, Amer. Math. Soc., Providence.

[8] R.Latala (2012) Moments of unconditional logarithmically concave vectors, in Geometric Aspects of Functional Analysis, Israel Seminar 2006-2010, 301-315, Lecture Notes in Math. 2050, Springer 2012.

[9] R.Latala (2014) Sudakov-type minoration for log-concave vectors, Studia Math. 223, 251-274.

[10] R. Latala and T. Tkocz (2015) A note on suprema of canonical processes based on random variables with regular moments, Electron. J. Probab. 20, 1-17.

[11] M. Ledoux And M. Talagrand (1991). Probability in Banach spaces. Isoperimetry and processes. Results in Math. and Rel. Areas (3). 23, Springer-Verlag, Berlin.

[12] M. B. Marcus and G. Pisier (1981) Random Fourier Series with Applications to Harmonic Analysis, Ann. of Math. Stud., 101, Princeton Univ. Press and Univ. Tokyo Press, Princeton, NJ and Tokyo.

[13] S. Mendelson and G. PaOuris (2012) On generic chaining and the smallest singular value of random matrices with heavy tails. J. Funct. Anal. 262, 3775-3811.

[14] S. Mendelson and R. Vershynin (2003) Entropy and the combinatorial dimension. Invent. Math. 152, 37-55.

[15] M. Talagrand (2014), Upper and Lower Bounds for Stochastic Processes. Modern Methods and Classical Problems. A Series of Modern Surveys in Mathematics, 60, Springer-Verlag, New-York. 